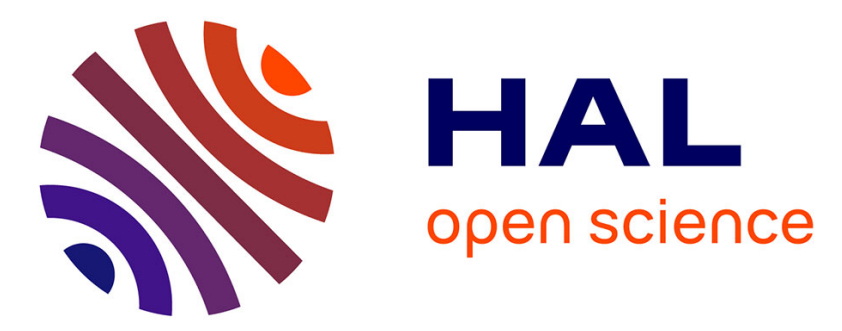

\title{
Microstructure of GaAs thin films grown on glass using Ge seed layers fabricated by aluminium induced crystallization
}

D. Pelati, G. Patriarche, L. Largeau, O. Mauguin, L. Travers, F. Brisset, F. Glas, F. Oehler

\section{To cite this version:}

D. Pelati, G. Patriarche, L. Largeau, O. Mauguin, L. Travers, et al.. Microstructure of GaAs thin films grown on glass using Ge seed layers fabricated by aluminium induced crystallization. Thin Solid Films, 2020, 694, pp.137737. 10.1016/j.tsf.2019.137737 . hal-02404458

\section{HAL Id: hal-02404458 https://hal.science/hal-02404458}

Submitted on 26 Nov 2020

HAL is a multi-disciplinary open access archive for the deposit and dissemination of scientific research documents, whether they are published or not. The documents may come from teaching and research institutions in France or abroad, or from public or private research centers.
L'archive ouverte pluridisciplinaire HAL, est destinée au dépôt et à la diffusion de documents scientifiques de niveau recherche, publiés ou non, émanant des établissements d'enseignement et de recherche français ou étrangers, des laboratoires publics ou privés. 


\title{
Microstructure of GaAs thin films grown on glass using Ge seed layers fabricated by Aluminium Induced Crystallization
}

\author{
D. Pelati ${ }^{\mathrm{a}, \mathrm{c}, \mathrm{d}}$, G. Patriarche ${ }^{\mathrm{a}}$, L. Largeau ${ }^{\mathrm{a}}$, O. Mauguin ${ }^{\mathrm{a}}$, L. Travers ${ }^{\mathrm{a}}$, F. Brisset ${ }^{\mathrm{b}}$, F. Glas ${ }^{\mathrm{a}}$, F. Oehler $^{\mathrm{a}, *}$ \\ ${ }^{a}$ Centre de Nanosciences et de Nanotechnologies, CNRS, Université Paris-Sud, Université Paris-Saclay, 10 Boulevard Thomas Gobert, 91120 \\ Palaiseau, France \\ ${ }^{b}$ Institut de Chimie Moléculaire et des Matériaux d'Orsay, CNRS, Université Paris-Sud, Université Paris-Saclay, 91405 Orsay, France \\ ${ }^{c}$ RIBER SA, 31 rue Casimir Périer, 95870 Bezons, France \\ ${ }^{d}$ Institut Photovoltaïque d'Ile-de-France (IPVF), 18 Boulevard Thomas Gobert, 91120 Palaiseau, France
}

\begin{abstract}
We perform the growth of GaAs epilayers by molecular beam epitaxy (MBE) on Ge pseudo-substrates obtained by the Aluminium Induced Crystallisation (AIC) of thin amorphous Ge layers deposited on silica. Despite the apparent uniformity of the AIC-Ge layer, large domains (more than $50 \mu \mathrm{m}$ wide) previously thought to be monocrystalline are found to actually consist in smaller grains (500 to $1000 \mathrm{~nm}$ wide), separated by low angle grain boundaries. These defects are transferred during epitaxy to the GaAs layer and degrade the quality of the III-V material. In our growth conditions, the MBE results in the selective deposition of thin GaAs layers on Ge with respect to the silica support, but selectivity is progressively lost with increasing layer thickness.
\end{abstract}

Keywords:

Metal Induced Crystallization, ALILE, Germanium, Aluminium, Gallium Arsenide, Molecular Beam Epitaxy

\section{Introduction}

Metal induced crystallization (MIC) is a promising technique for the fabrication of crystalline $\mathrm{Si}$ or Ge layers on low cost supports. Depending on the nature of the metal, the crystallization of amorphous Ge can occur at very low temperatures, down to $177^{\circ} \mathrm{C}$ for $\mathrm{Au}$ or $211^{\circ} \mathrm{C}$ for $\mathrm{Al}$, below the respective eutectic temperature of the binary mixtures $\left(\mathrm{Ge}-\mathrm{Al} 420^{\circ} \mathrm{C}\right.$, Ge-Au $\left.361^{\circ} \mathrm{C}\right)[1,2,3]$. The aluminium-induced crystallization (AIC) of Ge has attracted much interest due to the peculiar layer inversion process (ALILE) during the crystallization[4]. The low cost of the ALILE technique has primarily prompted solar applications $[5,6,7,8]$, although there have been other outcomes such as transistors $[9,10]$.

Interestingly, the control of the diffusion of $\mathrm{Ge}$ in the metal can lead to the crystallization of oriented grains with normals mainly along the [111] or [100] directions, depending on the nature of diffusion barrier and substrate[11, 12, 13, 14, 15, 16, 17]. The fabrication of flat oriented crystals with large grains Oehler)

Email address: fabrice.oehler@c2n.upsaclay.fr (F.

Preprint submitted to Thin Solid Films
21 (wider than $50 \mu \mathrm{m}$ ) is interesting and can compete 22 with other approaches such as localized liquid phase crystallization[18] or laser annealing[19, 20].

Yet, the use of MIC layers as oriented crystalline pseudo-substrates for subsequent epitaxy has been rather limited. Thin MIC-Si or MIC-Ge layers on silica have been used as substrates for the growth of GaAs[21, 22] or Ge[23] nanowires, but nanostructures with small footprints are not very demanding in terms of substrate crystallinity[24]. In an attempt to reduce defect density present in the MIC-Ge layer, Toko et al. performed the homoepitaxy of planar Ge layers on MIC-Ge pseudosubstrates by molecular beam epitaxy[15] (MBE). Due to the good lattice match between Ge and GaAs (lattice

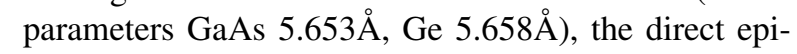
taxy of GaAs on such low-cost Ge layers is a promising route for the fabrication of cheaper III-V devices and solar cells. Comparable approaches have already been investigated in the literature, with GaAs solar cells grown on large-grain poly-Ge[25, 26] or Ge buffer on $\mathrm{Si}(100)[27]$. Recently, work by Toko et al. has shown a promising photoresponse from GaAs epilayers grown by MBE on MIC-Ge pseudo-substrates[28].

Yet the quality of MIC-Ge pseudo-substrates, which September 2, 2019 
consist in wide 'monocrystalline' domains[13, 15, 28], remains lower than commercial mono-crystalline $\mathrm{Ge}$ wafers. In particular no study has looked in details at the microstructure of the so-called 'mono-crystalline' MICGe domains and how it affects the subsequent GaAs epitaxy.

\section{Materials and methods}

We use here a fabrication procedure similar to that of Toko et al.[28]. A simplified scheme is presented Fig. 1 and highlights the two main steps: the fabrication of the MIC-Ge pseudo-substrates (Fig. 1.a) and the epitaxy of the GaAs epilayers (Fig. 1.b).

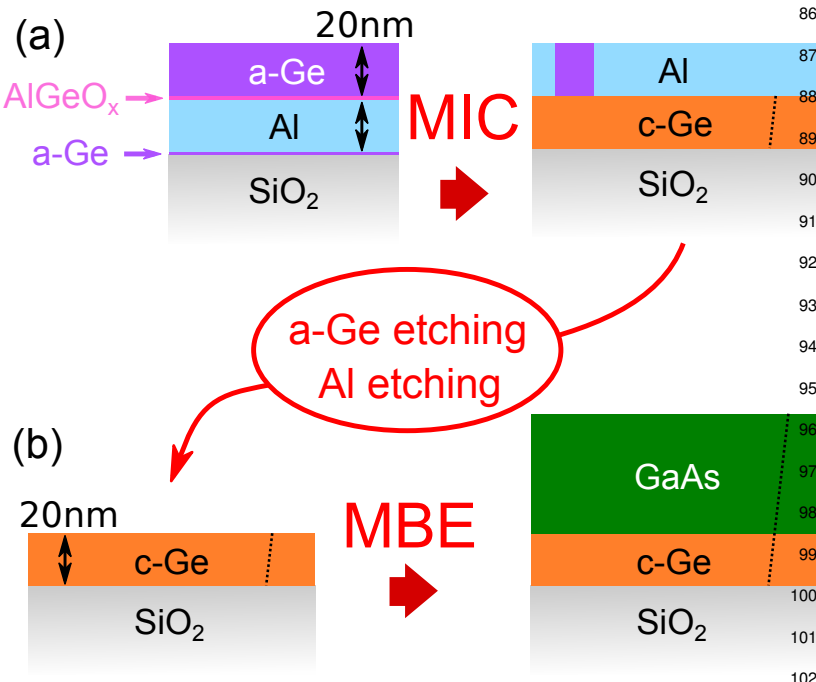

Figure 1: Simplified processing scheme of the samples. (a) Fabrication by MIC (ALILE) of the crystalline (c-Ge) pseudo-substrates starting from $20 \mathrm{~nm}$ thick amorphous $\mathrm{Ge}(\mathrm{a}-\mathrm{Ge})$ and aluminium layers deposited on silica. (b) Growth of GaAs on the Ge pseudo-substrates by molecular beam epitaxy (MBE).

The MIC-Ge pseudo-substrates are fabricated as follows. First, we form a $70 \mathrm{~nm}$ thick silica layer on standard $\mathrm{Si}(100)$ 2" wafers (Siltronix) by dry oxidation at $1050^{\circ} \mathrm{C}$ for $15 \mathrm{~min}$, which acts as a virtual amorphous silica substrate for the rest of the process. Samples fabricated from such oxidized $\mathrm{Si}$ wafers can be easily cleaved to fit the various equipments used in this study. Then, we deposit the Al and Ge layers by e-beam evaporation using a Plassys MEB550SL, as detailed in our previous work[29].

Before crystallization, the MIC-Ge sample consists 116 in a $1.0 \mathrm{~nm}$ Ge underlayer[30], followed by $20 \mathrm{~nm}$ of Al 117 and a $1.0 \mathrm{~nm}$ thick Ge layer which is exposed to air (for 118 $10 \mathrm{~min}$ ) to form a $\mathrm{AlGeO}_{\mathrm{x}}$ diffusion barrier[31, 32], and ${ }_{119}$ a final $20 \mathrm{~nm}$ thick Ge top layer. The MIC is performed in a programmable hot plate (Harry Gestigkeit) flushed with nitrogen gas by heating the sample at $270-300{ }^{\circ} \mathrm{C}$ during 15 to $20 \mathrm{~h}$. After the annealing, the remaining amorphous $\mathrm{Ge}$ is selectively removed using reactive ion etching (CCP/RIE Nextral NE100, using $\mathrm{O}_{2}$ and $\mathrm{SF}_{6}$ for $4 \mathrm{~min}$ [33]. Then the top Al layer is removed chemically using $\mathrm{H}_{3} \mathrm{PO}_{4}$ at $100^{\circ} \mathrm{C}$ during approximately $60 \mathrm{~s}$. The change of the surface reflectivity, from the bright Al layer to the darker crystalline Ge material (c-Ge), is used to control the wet etch duration.

Before introduction in the Molecular Beam Epitaxy (MBE) chamber (RIBER 32) the MIC-Ge pseudosubstrates are further cleaned using $\mathrm{H}_{2} \mathrm{O}_{2}(30 \mathrm{~s})$ and $\mathrm{HF} 1 \%(30 \mathrm{~s})$ treatments, with intermediate rinse in deionized water. The MIC-Ge substrates are then outgassed at $400^{\circ} \mathrm{C}$ in a dedicated ultra high vacuum chamber, before transfer to the main growth chamber (base pressure $1.010^{-10}$ Torrs). The GaAs epitaxy is performed using parameters previously optimized for the twin-free epitaxy of GaAs(111) on Ge(111) monocrystalline wafers[34].

Here, gallium is provided by a standard effusion cell, while arsenic is delivered by a valved cracker cell (RIBER VAC500) producing $\mathrm{As}_{4}$ tetramers (cracker temperature $600^{\circ} \mathrm{C}$ ). The $\mathrm{Ga}$ beam equivalent pressure (BEP) is set to $1.510^{-7}$ Torrs, corresponding to a growth rate of $\sim 1.5 \AA . \mathrm{s}^{-1}$ on GaAs (100). The ratio of arsenic to gallium $\left(\mathrm{BEP}_{\mathrm{As}_{4}}: \mathrm{BEP}_{\mathrm{Ga}}\right)$ is approximately $60: 1$. The substrate temperature is $625-635^{\circ} \mathrm{C}$, as measured by thermocouple and optical pyrometry, during all the growth procedure.

The samples are characterized before and after growth using Scanning Electron Microscopy (SEM, FEI Magellan). The local crystalline orientation is measured using Electron Backscatter Diffraction (EBSD, ZEISS Supra 55 VP with Hikari/OIM TSL EDAX detector). The layers are also characterized by Transmission Electron Microscopy (TEM, FEI Titan THEMIS) using Energy Dispersive X-ray Spectroscopy (EDX, Bruker Super-X). Focused Ion Beam (FIB, FEI Helios) is used to prepare the TEM foils.

\section{Results}

\subsection{Growth}

Figure 2 presents the surface morphology before and after the GaAs growth. The initial MIC-Ge surface is shown Fig. 2.a, in which the silica support appears dark. The growth of a $40 \mathrm{~nm}$ thick GaAs layer (at $630^{\circ} \mathrm{C}$ for $4.5 \mathrm{~min}$ ) creates large density of triangular terraces and 

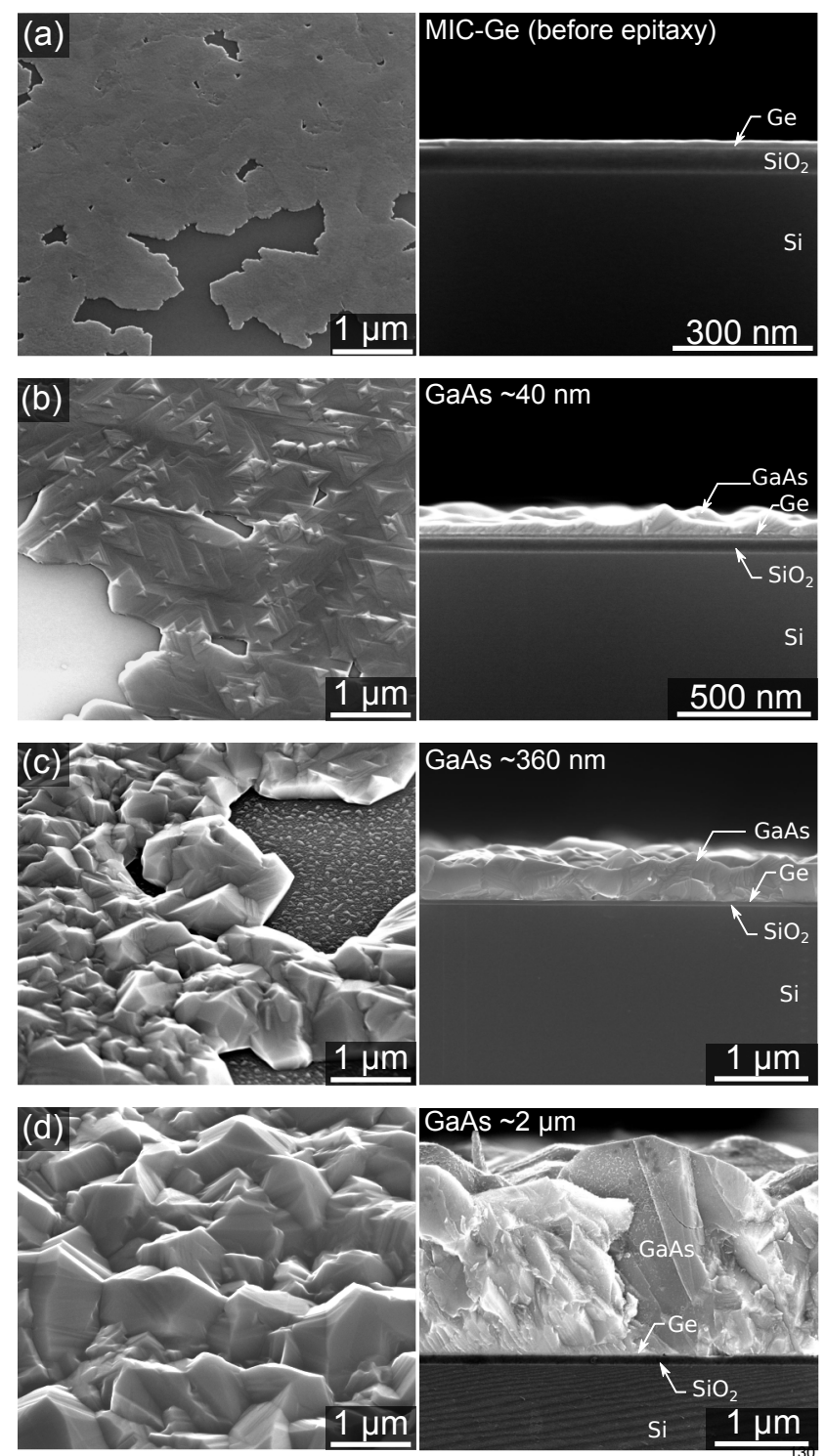

Figure 2: SEM image of the samples before and after GaAs growth of increasing thickness. (a) Bird's eye view ( $45^{\circ}$ tilt) and cross sectional 13 image of the initial MIC-Ge layer. (b-d) Similar images after $40 \mathrm{~nm},{ }_{132}$ $360 \mathrm{~nm}$ and $2 \mu \mathrm{m}$ thick GaAs growth on MIC-Ge pseudo-substrates.
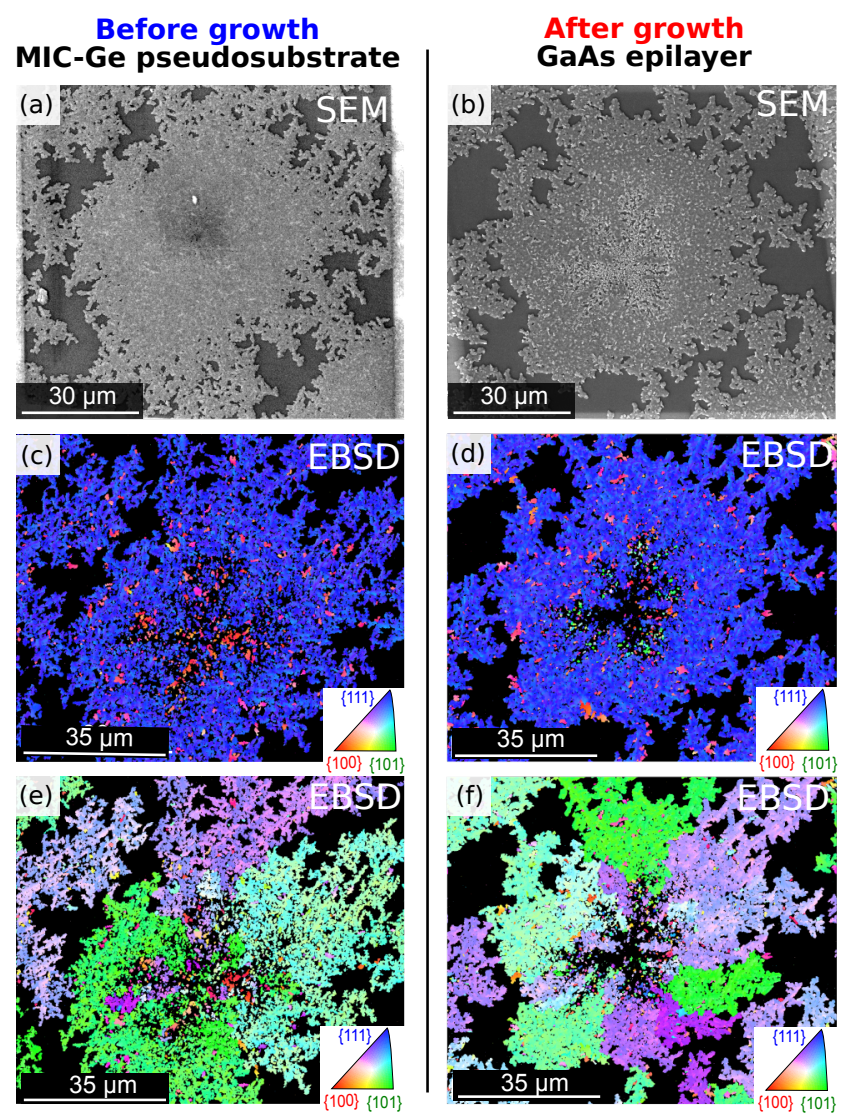

Figure 3: Different zones from the initial MIC-Ge pseudo-substrate (before growth, left) and the GaAs epilayer (after growth, right). (a)(b) SEM top views of the MIC-Ge and GaAs surfaces, respectively. (c)(d) Corresponding ESBD maps of the out of plane crystal orientation. The color is assigned from the inverse pole figure in insert. (e)(f) Corresponding EBSD maps of the in plane crystal orientation. Same color scale as in (c-d).

(Fig. 2.d).

\subsection{Crystal orientation}

Figure 3 presents SEM and EBSD characterizations of the sample before and after $40 \mathrm{~nm}$ thick GaAs growth. Before growth (Fig. 3.a) MIC-Ge pseudosubstrate is structured in large $\mathrm{Ge}$ islands on $\mathrm{SiO}_{2}$ (more than $50 \mu \mathrm{m}$ diameter, $20 \mathrm{~nm}$ thick) which are mostly (111) oriented, as indicated by the dominant blue color in the out of plane EBSD (Fig. 3.c). Although the island shown in Fig. 3.a appears homogeneous, it is actually made of three separate dendritic crystals with different in plane orientations (Fig. 3.e). The central core is a defective zone with mixed orientation[29]. Due to the small grain size and poor crystal quality in this area, the corresponding zones appears black in the EBSD maps, Fig. 3.c and 3.e. Between the Ge islands, the amorphous 


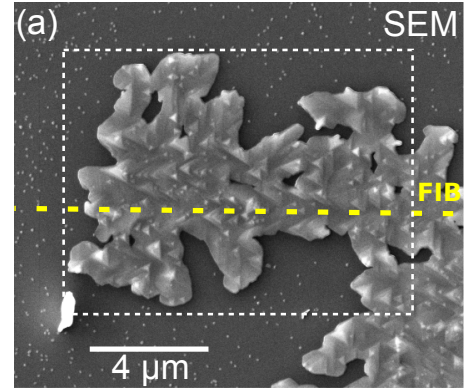

(b)
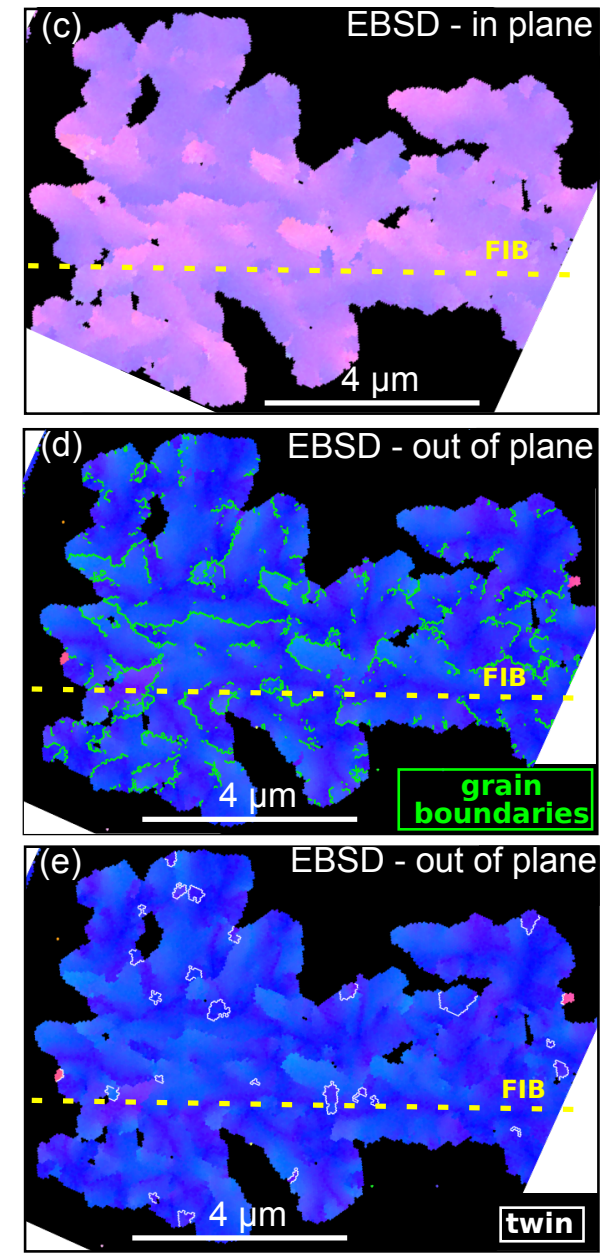

Figure 4: SEM and EBSD characterizations of an area with uniform 187 in plane orientation. The yellow dashed line marks the position of the FIB cut used to extract the TEM foil. (a) Top view SEM image showing the zone selected for EBSD analysis (white rectangle). (b) Inverse pole figure (IPF) color scheme used in the EBSD maps.(c) 190 EBSD map of the in plane crystal orientation. (d) Out of plane EBSD 191 map showing grain boundaries $\left(0-10^{\circ}\right)$ in green. (e) Out of plane crystal orientation, twin boundaries are marked in white. also appears black on the EBSD maps.
Figure 3.b presents the morphology after the growth of a thin GaAs layer $(40 \mathrm{~nm})$. As already seen in 150 Fig. 2.b, this short GaAs deposition is selective with re51 spect to the silica support and the GaAs crystals only grow on the MIC-Ge islands. EBSD characterization (Fig. 3.d) shows that the preferential (111) orientation of the MIC-Ge layer (Fig. 3.c, blue color) is conserved in the GaAs layer. The central defective core and the dendritic crystals with different in plane orientations are also visible in the GaAs crystal orientation (Fig. 3.f), as observed in the MIC-Ge layer before growth (Fig. 3.e).

By zooming on the termination of a large dendrite (Fig. 4.a) we can focus on an apparently monocrystalline area with uniform in plane orientation (Fig. 4.c) and (111) crystal orientation (Fig. 4.d). At this scale, small correlated color in the in plane and out of plane EBSD maps shifts hint at the presence of small adjacent grains with slightly different crystal orientation. To reveal this grainy structure, we highlight in bright green local misorientations $\left(0-10^{\circ}\right)$ between neighboring points (Fig. 4.d). The obtained set of grain boundaries drastically reduces the typical grain size from several tens of microns to one micron approximately. Figure 4.e highlights in white color another set of misorientation $\left(60^{\circ}\right.$ in plane rotation), which corresponds to twinned domains for (111)-oriented cubic crystals. The typical lateral size of twinned domains is also of the order of one micron or smaller.

\subsection{Microstructure}

To better understand the microstructure of the MICGe and GaAs layers, we perform a FIB cut in the previously analyzed 'monocrystalline' area (see yellow dashed line in Fig. 4). Figure 5 shows $g_{002}$ dark field images from the TEM cross section oriented along the

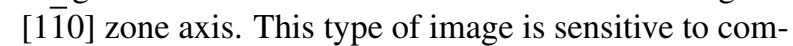
positional changes and permits to differentiate GaAs from and $\mathrm{Ge}$, despite the close lattice match and crystal structure.

In a defect free region (Fig. 5.b), we observe a smooth MIC-Ge layer with a thickness of $20 \mathrm{~nm}$, which is equal to the nominal thickness of the initial amorphous Ge layer (see methods). The thickness of the GaAs layer is $40 \mathrm{~nm}$, as expected from the MBE growth rate (1.5 ̊.. $\mathrm{s}^{-1}$ ) and duration (4.5 min).

While no defect is visible in this smooth area (Fig. 5.b), pyramidal hillocks and vertical defects are found in zone with thicker $(\sim 60 \mathrm{~nm})$ GaAs (Fig. 5.c). These defects originate from the MIC-Ge layer (orange arrow) and extend to the whole GaAs thickness (green arrows). The area bordering these vertical defect are 


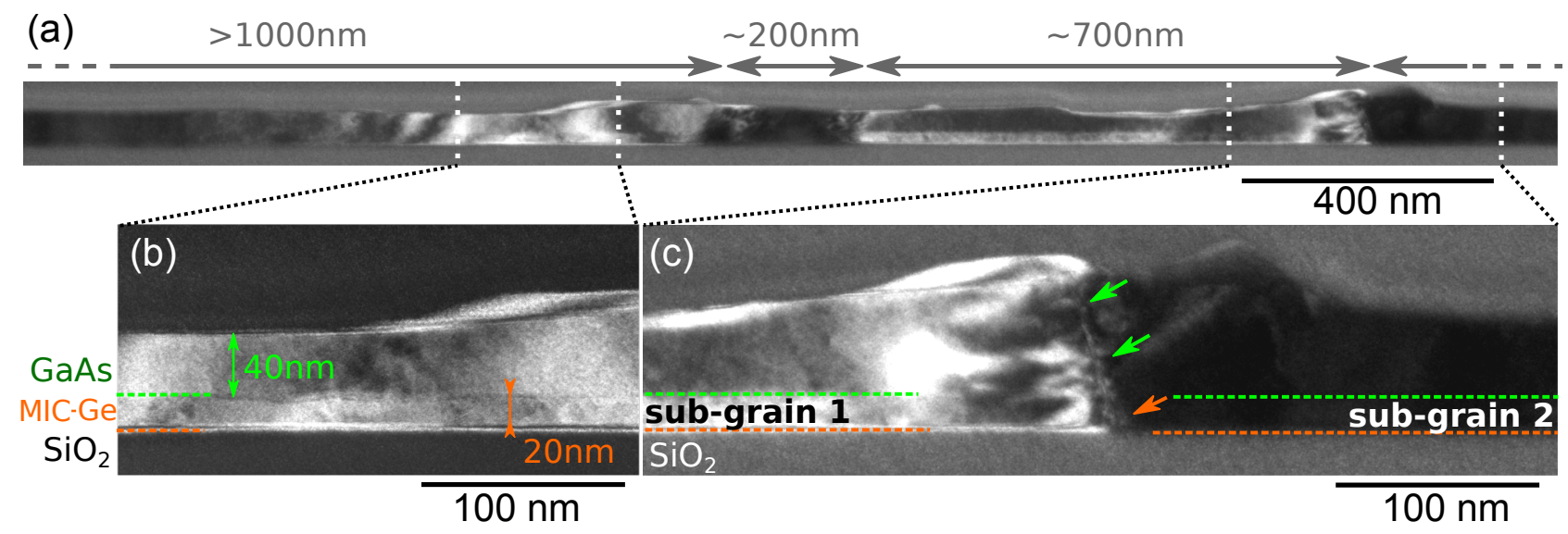

Figure 5: Dark field cross-sectional $g_{002}$ TEM image of the GaAs / MIC-Ge pseudo-substrate near the [1 $1 \overline{1} 0$ ] zone axis. (a) Large scale view showing the zones selected for further analysis. The lateral size of the sub-grains is indicated above the image. (b) Details of a smooth area showing the thickness of each layer on the $\mathrm{SiO}_{2}$ support. (c) Details of a pyramidal hillock. A defect from the MIC-Ge pseudo-substrate (orange arrow) extends to the GaAs layer (green arrows).

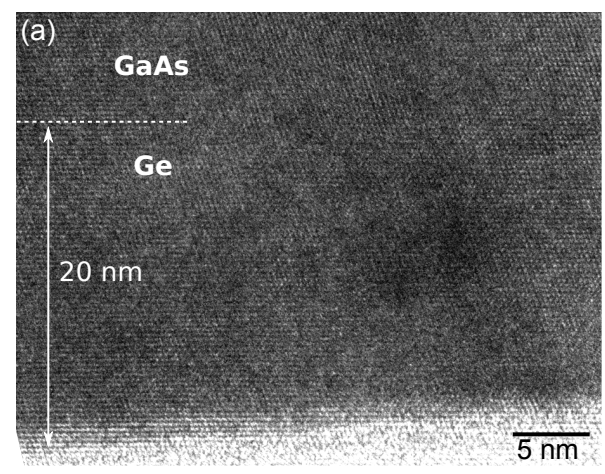

(b)

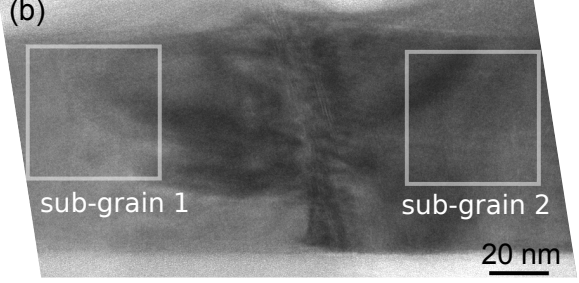

$(\mathrm{C})$

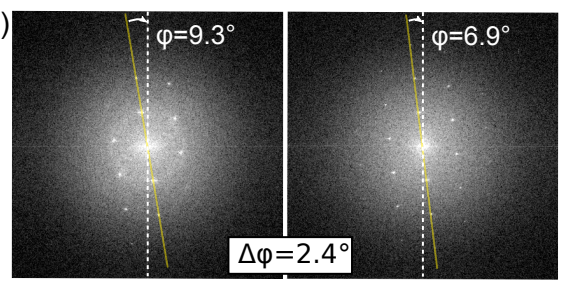

Figure 6: HR-TEM images of the zones analyzed in Fig. 5. (a) Atom- 219 ically resolved image of the smooth area (see Fig. 5.b). (b) Image of 220 the defective zone with two sub-grains (see Fig. 5.c). The left and 221 right squares mark the zones used to perform FFT. (c) FFT patterns of the left and right sub-grain. Both GaAs crystals are cubic with a relative angular misalignment of $\Delta \varphi=2.4^{\circ}$. highly contrasted. Sub-grain \#1 (left of the defect) appears in light color while sub-grain \#2 (at right) is dark. The lateral size of the sub-grains is between 200 and 1000 nm, or possibly larger (see Fig. 5.a).

Figure 6 presents high resolution TEM (HR-TEM) images of the zones previously analyzed in Fig. 5. The smooth area is detailed in Fig. 6.a. There, we observe the continuation of the cubic crystal lattice from the MIC-Ge pseudo-substrate to the GaAs layer. Figure 6.b shows HR-TEM image of the defective area of Fig. 5.c - Two zones are selected in this image to compute a fast Fourier transform (FFT). This FFT (Fig. 6.c) reveals that two standard cubic patterns with a relative misorientation of $\Delta \varphi=2.4^{\circ}$ between the two crystals. This demonstrates that the extended defect of Fig. 5.c and Fig. 6.b is a low-angle grain boundary (LAGB).

\section{Discussion}

Beside a few reports on nanowire growth[21, 22, 23], we could only find an two attempts at planar layer epitaxy on MIC substrates: homoepitaxy of Ge on MIC-Ge(111)[15] and hetero-epitaxy of GaAs on MICGe(111)[28]. Contrary to Toko et al.[28], who report good photoresponse from GaAs layers grown on MIC$\mathrm{Ge}$, the disrupted morphology of our thick GaAs layers (Fig. 2) indicates that defects degrades the III-V material quality, at length scales much smaller to the apparent domain size (over $20 \mu \mathrm{m}$ ) determined by large scale EBSD (Fig. 3). 


\subsection{Absence of antiphase boundaries}

Compared to commercial 'epiready' monocrystalline Ge wafers, the surface of our MIC-Ge pseudo-substrates is very rough: nanometer sized variations of the MICGe thickness can be seen directly by cross-sectional TEM in Fig. 5, even in the defect-free area. Due to the small thickness of the MIC-Ge layer $(20 \mathrm{~nm})$ further chemical or mechanical polishing is proscribed. It is thus critical that the GaAs epitaxy is not sensitive to the Ge surface roughness. Yet, even the standard epitaxy case of polar GaAs on non-polar $\mathrm{Ge}$ is known to require a carefully reconstructed double-step $\mathrm{Ge}(100)$ surface and a specific wafer miscut[35, 36]. The direct GaAs epitaxy on (100)-oriented MIC-Ge layers is thus likely to hold high densities of antiphase boundaries (APB).

The identification of APB from EBSD maps is a difficult task, as the standard IPF color scheme (Fig. 4.b) is insensitive to $180^{\circ}$ rotations, so that it cannot discriminate grains with opposite orientations. Note that this applies to both out of plane (Fig. 4.d) and in plane (Fig. 4.c) EBSD maps.

Fortunately, the EBSD data (Fig. 3 and 4) show that the main orientation of our MIC-Ge layers is (111), similarly to most layers fabricated by ALILE on silica[13, 30, 15]. On commercial 'epiready' Ge(111) wafers, the polarity of GaAs epilayers grown by MBE is uniquely determined and insensitive to the surface roughness, so that no antiphase domain form during the growth[37, 34]. Our TEM analysis (Fig. 5) did not reveal any APB in our GaAs layers, despite the lower surface quality of our MIC-Ge pseudo-substrates compared to commercial $\mathrm{Ge}(111)$ wafers. The growth of $\mathrm{GaAs}$ on (111)-oriented MIC-Ge templates remains ABP-free and it is thus a promising combination for the fabrication of low-cost III-V devices on silica.

\subsection{Selective growth of GaAs on MIC-Ge(111)/SiO}

The use of a silica support for the MIC-Ge layers is particularly relevant since $\mathrm{SiO}_{2}$ masks are already used in the selective area growth of $\mathrm{Ge}[38]$ or $\mathrm{GaAs}[39,40]$ by MBE. Despite the relatively high substrate temperature $\left(\sim 630^{\circ} \mathrm{C}\right)$, we only observe a partial selectivity for the GaAs growth between the MIC-Ge islands and the $\mathrm{SiO}_{2}$ support, which is progressively lost as the deposition duration increases from 4.5 to $40 \mathrm{~min}$ (Fig. 2). It is possible that the exposed silica still host some residual $\mathrm{Al}$ or Ge contaminations from the deposition of the initial MIC structure, despite the thorough chemical cleaning of the MIC-Ge layers (see methods). A solution to this partial selectivity issue is to fabricate MIC-Ge layers with a high surface coverage $[13,15]$ so that no $\mathrm{SiO}_{2}$ is exposed to GaAs during the MBE growth[28].

\section{4.3. Epitaxial relationship GaAs/MIC-Ge(111)}

By focusing on the thin GaAs layer $(\sim 40 \mathrm{~nm})$ for which the growth is still fully selective (Fig. 2), we can learn more about the epitaxial relationship between the GaAs layer and the MIC-Ge pseudo-substrate. The transfer of the large scale (111) crystal texture from the MIC-Ge template to the GaAs epilayer (Fig. 3) is a good indication of the epitaxy relationship. This hypothesis is confirmed by cross-sectional HR-TEM images (Fig. 6), where we observe the continuation of the crystal lattice from the cubic MIC-Ge grain to the GaAs cubic crystal. The growth of GaAs (111) on the MIC$\mathrm{Ge}(111)$ thus proceeds as on standard monocrystalline Ge substrates[37, 41,34] and preserve in GaAs the same in plane and out of plane orientations as the Ge substrate. The absence of visible defect by TEM at the $\mathrm{GaAs} / \mathrm{Ge}(111)$ interface (Fig. 5) demonstrates that our substrate fabrication and cleaning procedures enable the defect-free epitaxy of GaAs(111) on MIC-Ge(111).

\subsection{Twinning in GaAs(111) epilayers}

As the GaAs growth proceeds in epitaxy with the MIC-Ge substrate, it seems likely that the crystal orientation of MIC-Ge grains is exactly that of GaAs epilayer. Yet it is possible that extended defects in the GaAs layer alter this initial epitaxy relationship.

The color scheme of the EBSD maps stems from a standard IPF (Fig. 4.b). As stated before, this color coding does not differentiate between $180^{\circ}$ rotations, so that it is not only insensitive to antiphase domains but also to twin defects. However twin boundaries can be revealed by showing the local $60^{\circ}$ in plane misorientations in (111)-oriented domains (Fig. 4.e).

From previous work[34] and literature[41] using commercial monocrystalline $\mathrm{Ge}(111)$ wafers, we know that the MBE growth of GaAs on $\mathrm{Ge}(111)$ is prone to twinning and that the twin density can be minimized by adapting the V:III ratio during growth. The Figure 4.e shows that twinned area fraction is relatively low (3\%) using MIC-Ge pseudo-substrates. Yet, we cannot fully replicate the growth of twin-free GaAs layers obtained on commercial Ge(111) wafers, even we use the same optimized growth conditions[34]. It is also possible that twinning occurs in the MIC-Ge layer, during the Ge crystallization, but we did no investigate this hypothesis further due to the low density of twinned domains compared to other extended defects.

\subsection{Microsctructure of MIC-Ge pseudo-substrates}

We previously studied the macrostructure of our MIC-Ge layers on silica using in situ optical 
microscopy[29]. This study showed that the defec- 378 tive core of each Ge island crystallizes from a para- 379 sitic MIC process, while the radially expanding (111)- 380 oriented dendrites are created by the standard ALILE 381 mechanism. These large Ge have different in plane ori- 382 entations (Fig. 3.e), and it is generally accepted that they 383 are monocrystalline[15, 30, 31], as supported by large 384 scale EBSD maps.

However, one can usually find grains with a differ- 386 ent orientation, typically close to (100), in the other- 387 wise (111)-oriented dendritic crystal (see red dots vs 388 blue patches in Fig. 3.c). By zooming on the termination 389 of a large dendrite (Fig. 4), we avoid such localized de- 390 fects and we can assess the quality of the main fraction 391 of the GaAs epilayer. Hence, whereas the GaAs appears homogeneous at large scale, it breaks down into small sub-micronic domains at small scale. Beside a 392 minor fraction of twinned domains (Fig. 4.e), we observe correlated color shifts in the in plane (Fig. 4.c) 393 and out of plane (Fig. 4.d) EBSD projections, some 394 of which are identified as grain boundaries (Fig. 4.d). 395 However EBSD characterization is usually performed 396 on flat surfaces, which are usually obtained after fine 397 mechanical polishing or ion milling. Considering the 398 disrupted morphology of our $40 \mathrm{~nm}$ thick GaAs layers 399 (Fig. 4.a), the small angular misorientations highlighted 400 in Fig. 4.d could be EBSD artefacts due to rough surface 401 of the sample. It is therefore important to complement 402 the EBSD data by another characterization technique. $\quad 403$

Using cross-sectional TEM, we link the position of 404 the triangular hillocks observed by SEM (Fig. 4.a) 405 with those of extended defects inside the GaAs layer 406 (Fig. 5). Further analysis (Fig. 6) demonstrates that the 407 observed extended defects are low angle grain bound- 408 aries (LAGBs). Combining both TEM analyses (Fig. 5- 409 6) with the SEM overview (Fig. 4), we hypothesize that 410 each hillock may be associated to a LAGB. This high 411 density of LAGB is coherent with the estimated position 412 of grain boundaries computed by EBSD (Fig. 4.d). The ${ }_{413}$ typical grain size determined by TEM, (200-1000 nm, 414 Fig. 5) matches the length scale of the small tonal 415 changes in the EBSD maps (Fig. 4.c) and the sub-grain width of Fig. 4.d. The inner structure of a LAGB consisting in a dense 1D array of dislocations[42], the high 416 density of LAGBs also explains the poor optical quality of our GaAs epilayers (not shown).

The Fig. 5.a and Fig. 6.b clearly show that the LAGB ${ }_{418}$ originates from the MIC-Ge layer. This implies that 419 the epitaxial growth merely extends the defective mi- 420 crostructure of the MIC-Ge pseudo-substrate to the ${ }_{421}$ GaAs layer. Compared to other attempt at planar epi- 422 taxy using MIC-Ge pseudo-substrates[15, 28], we ob- 423 serve defect-free regions (MIC-Ge and GaAs layers) which span more than several hundreds of nanometer (Fig. 5), with only a couple of LAGB over one micron. This suggests that the quality of our MIC-Ge material is better if not comparable to the state of the art.

Moreover, we note that the small orientation shifts characterized by EBSD (Fig. 4), which we link to the defective LAGB microstructure (Fig 6), are visible in most of the literature on crystalline Ge layers fabricated using ALILE[15, 30, 31, 13] or gold-induced crystallization[43]. It is thus likely that the formation of LAGB is intrinsic to the metal-assisted crystallization of thin Ge layers, as regards the published results on (111)-oriented MIC-Ge layers on silica.

\section{Conclusion}

We have successfully performed the heteroepitaxy of GaAs by MBE on (111)-oriented MIC-Ge pseudosubstrates fabricated on $\mathrm{SiO}_{2}$ supports. The epitaxy relationship between GaAs and MIC-Ge is the same as for standard growth on monocrystalline bulk Ge wafers. No extended defect nucleates at the GaAs/Ge growth interface, which indicates that our cleaning procedures are adequate. The low fraction of twinned GaAs crystals indicates that our MBE growth conditions are close to optimal values. However, the epitaxial growth transfers the defective microstructure of the MIC-Ge pseudosubstrate to the GaAs epilayer so that the final III-V material is of poor quality. Despite an apparent homogeneity at large scale and EBSD maps similar to the current literature, we find that our MIC-Ge layers are composed of slightly misaligned small crystallites (200 to $1000 \mathrm{~nm}$ wide), even in apparently 'monocrystalline' domains. This defective microstructure appears as small tonal variations in standard EBSD maps. The observed high density of low-angle grain boundaries, hence dense dislocation arrays, impact the final III-V material quality and may limit the performance of III-V devices fabricated on MIC-Ge pseudo-substrates.

\section{Acknowledgement}

The authors thank the Institut Photovoltaique d'Ile de France (IPVF) for financial support under framework project E.3. The authors also acknowledge ANR Investissement d'Avenir program (TEMPOS Project ANR-10-EQPX-50) for having funded the acquisition of the NANOTEM platform and the TEM-STEM (FEI Titan Themis) used in this work. 


\section{References}

[1] S. Gaudet, C. Detavernier, A. J. Kellock, P. Desjardins, C. Lavoie, Thin film reaction of transition metals with germanium, Journal of Vacuum Science \& Technology A 24 (3) (2006) 474-485 (2006). doi:10.1116/1.2191861.

[2] W. Knaepen, C. Detavernier, R. V. Meirhaeghe, J. J. Sweet, C. Lavoie, In-situ X-ray diffraction study of metal induced crystallization of amorphous silicon, Thin Solid Films 516 (15) (2008) 4946-4952 (2008). doi:10.1016/j.tsf.2007.09.037.

[3] W. Knaepen, S. Gaudet, C. Detavernier, R. L. V. Meirhaeghe, J. J. Sweet, C. Lavoie, In situ x-ray diffraction study of metal induced crystallization of amorphous germanium, Journal of Applied Physics 105 (8) (2009) 083532 (2009). doi:10.1063/1.3110722

[4] O. Nast, S. R. Wenham, Elucidation of the layer exchange mechanism in the formation of polycrystalline silicon by aluminuminduced crystallization, Journal of Applied Physics 88 (1) (2000) 124-132 (2000). doi:10.1063/1.373632.

[5] O. Nast, S. Brehme, S. Pritchard, A. G. Aberle, S. R. Wenham, Aluminium-induced crystallisation of silicon on glass for thinfilm solar cells, Solar Energy Materials and Solar Cells 65 (1-4) (2001) 385-392 (2001). doi:10.1016/s0927-0248(00)00117-3.

[6] I. Gordon, D. V. Gestel, K. V. Nieuwenhuysen, L. Carnel, G. Beaucarne, J. Poortmans, Thin-film polycrystalline silicon solar cells on ceramic substrates by aluminium-induced crystallization, Thin Solid Films 487 (1-2) (2005) 113-117 (2005). doi:10.1016/j.tsf.2005.01.047.

[7] D. V. Gestel, I. Gordon, J. Poortmans, Aluminum-induced crystallization for thin-film polycrystalline silicon solar cells: Achievements and perspective, Solar Energy 5 Materials and Solar Cells 119 (2013) 261-270 (2013). doi:10.1016/j.solmat.2013.08.014.

[8] O. Shekoofa, J. Wang, D. Li, Y. Luo, C. Sun, Z. Hao, ${ }^{521}$ Y. Han, B. Xiong, L. Wang, H. Li, P-silicon thin film fabricated by magnetron sputtering and aluminium induced ${ }^{523}$ crystallization for schottky silicon solar cells, Materials Sci- ${ }^{524}$ ence in Semiconductor Processing 71 (2017) 366-373 (2017). doi:10.1016/j.mssp.2017.06.008.

[9] R. Chen, W. Zhou, M. Zhang, M. Wong, H.-S. Kwok, Highperformance polycrystalline silicon thin-film transistors based on metal-induced crystallization in an oxidizing atmosphere, IEEE Electron Device Letters 36 (5) (2015) 460-462 (2015). doi: $10.1109 /$ led.2015.2409858.

10] K. Moto, K. Yamamoto, T. Imajo, T. Suemasu, H. Nakashima, K. Toko, Polycrystalline thin-film transistors fabricated on highmobility solid-phase-crystallized Ge on glass, Applied Physics Letters 114 (21) (2019) 212107 (2019). doi:10.1063/1.5093952.

[11] A. Okada, K. Toko, K. O. Hara, N. Usami, T. Suemasu, Dependence of crystal orientation in Al-induced crystallized poly-Si layers on $\mathrm{SiO} 2$ insertion layer thickness, Journal of Crystal Growth 356 (2012) 65-69 (2012). doi:10.1016/j.jcrysgro.2012.07.015.

[12] R. Numata, K. Toko, N. Saitoh, N. Yoshizawa, N. Usami, T. Suemasu, Orientation control of large-grained Si films on insulators by thickness-modulated Al-induced crystallization, Crystal Growth \& Design 13 (4) (2013) 1767-1770 (2013). doi:10.1021/cg4000878.

[13] K. Nakazawa, K. Toko, N. Saitoh, N. Yoshizawa, N. Usami, ${ }^{546}$ T. Suemasu, Large-grained polycrystalline (111) Ge films on in- ${ }^{547}$ sulators by thickness-controlled Al-induced crystallization, ECS Journal of Solid State Science and Technology 2 (11) (2013) Q195-Q199 (2013). doi:10.1149/2.007311jss.

[14] K. Toko, R. Numata, N. Saitoh, N. Yoshizawa, N. Usami, 55 T. Suemasu, Selective formation of large-grained, (100)- or ${ }^{552}$ (111)-oriented $\mathrm{Si}$ on glass by Al-induced layer exchange, Journal of Applied Physics 115 (9) (2014) 094301 (2014). doi:10.1063/1.4867218

[15] K. Toko, K. Nakazawa, N. Saitoh, N. Yoshizawa, T. Suemasu, Improved surface quality of the metal-induced crystallized Ge seed layer and its influence on subsequent epitaxy, Crystal Growth \& Design 15 (3) (2015) 1535-1539 (2015). doi:10.1021/acs.cgd.5b00060.

[16] J.-H. Park, T. Suzuki, M. Kurosawa, M. Miyao, T. Sadoh, Nucleation-controlled gold-induced-crystallization for selective formation of $\mathrm{Ge}(100)$ and (111) on insulator at low-temperature (250C), Applied Physics Letters 103 (8) (2013) 082102 (2013). doi:10.1063/1.4819015.

[17] N. Oya, K. Toko, N. Saitoh, N. Yoshizawa, T. Suemasu, Effects of flexible substrate thickness on Al-induced crystallization of amorphous Ge thin films, Thin Solid Films 583 (2015) 221-225 (2015). doi:10.1016/j.tsf.2015.03.072

[18] S. Hu, P. W. Leu, A. F. Marshall, P. C. McIntyre, Singlecrystal germanium layers grown on silicon by nanowire seeding, Nature Nanotechnology 4 (10) (2009) 649-653 (2009). doi:10.1038/nnano.2009.233.

[19] S. Kühnapfel, S. Gall, B. Rech, D. Amkreutz, Towards monocrystalline silicon thin films grown on glass by liquid phase crystallization, Solar Energy Materials and Solar Cells 140 (2015) 86-91 (2015). doi:10.1016/j.solmat.2015.03.030.

[20] K. Toko, T. Tanaka, T. Sadoh, M. Miyao, Formation of singlecrystalline Ge stripes on quartz substrates by SiGe mixingtriggered liquid-phase epitaxy, Thin Solid Films 518 (6) (2010) S179-S181 (2010). doi:10.1016/j.tsf.2009.10.083.

[21] Y. Cohin, O. Mauguin, L. Largeau, G. Patriarche, F. Glas, E. Søndergård, J.-C. Harmand, Growth of vertical GaAs nanowires on an amorphous substrate via a fiber-textured Si platform, Nano Letters 13 (6) (2013) 2743-2747 (2013). doi:10.1021/nl400924c.

[22] D. Ren, I. M. Høiaas, J. F. Reinertsen, D. L. Dheeraj, A. M. Munshi, D.-C. Kim, H. Weman, B.-O. Fimland, Growth optimization for self-catalyzed GaAs-based nanowires on metalinduced crystallized amorphous substrate, Journal of Vacuum Science \& Technology B, Nanotechnology and Microelectronics: Materials, Processing, Measurement, and Phenomena 34 (2) (2016) 02L117 (2016). doi:10.1116/1.4943926.

[23] K. Toko, M. Nakata, W. Jevasuwan, N. Fukata, T. Suemasu, Vertically aligned Ge nanowires on flexible plastic films synthesized by (111)-oriented Ge seeded vapor-liquid-solid growth, ACS Applied Materials \& Interfaces 7 (32) (2015) 1812018124 (2015). doi:10.1021/acsami.5b05394.

[24] V. Dhaka, T. Haggren, H. Jussila, H. Jiang, E. Kauppinen, T. Huhtio, M. Sopanen, H. Lipsanen, High quality GaAs nanowires grown on glass substrates, Nano Letters 12 (4) (2012) 1912-1918 (2012). doi:10.1021/nl204314z.

[25] M. G. Mauk, Low-cost III-V compound semiconductor solar cells, in: Handbook of Research on Solar Energy Systems and Technologies, IGI Global, 2012, pp. 254-293 (2012). doi:10.4018/978-1-4666-1996-8.ch010.

[26] R. Venkatasubramanian, B. C. O. Quinn, E. Siivola, B. Keyes, R. Ahrenkiel, 20\% (AM1.5) efficiency GaAs solar cells on sub$\mathrm{mm}$ grain-size poly-Ge and its transition to low-cost substrates, in: Conference Record of the Twenty Sixth IEEE Photovoltaic Specialists Conference - 1997, IEEE, 1997, pp. 811-814 (1997). doi:10.1109/pvsc. 1997.654211.

[27] Y. Wang, Z. Ren, M. Thway, K. Lee, S. F. Yoon, I. M. Peters, T. Buonassisi, E. A. Fizgerald, C. S. Tan, K. H. Lee, Fabrication and characterization of single junction GaAs solar cells on $\mathrm{Si}$ with As-doped $\mathrm{Ge}$ buffer, Solar Energy Materials and Solar Cells 172 (2017) 140-144 (2017). 
doi:10.1016/j.solmat.2017.07.028

28] T. Nishida, K. Moto, N. Saitoh, N. Yoshizawa, T. Suemasu, 610 K. Toko, High photoresponsivity in a GaAs film synthe- 620 sized on glass using a pseudo-single-crystal Ge seed layer, 621 Applied Physics Letters 114 (14) (2019) 142103 (2019). 622 doi:10.1063/1.5091714.

[29] D. Pelati, G. Patriarche, O. Mauguin, L. Largeau, F. Brisset, 624 F. Glas, F. Oehler, In situ optical monitoring of new path- 625 ways in the metal-induced crystallization of amorphous Ge, 626 Crystal Growth \& Design 17 (11) (2017) 5783-5789 (2017). 627 doi:10.1021/acs.cgd.7b00799.

[30] R. Numata, K. Toko, K. Nakazawa, N. Usami, T. Suemasu, Growth promotion of Al-induced crystallized Ge films on insulators by insertion of a $\mathrm{Ge}$ membrane below the Al layer, Thin Solid Films 557 (2014) 143-146 (2014). doi:10.1016/j.tsf.2013.08.040.

[31] R. Numata, K. Toko, N. Oya, N. Usami, T. Suemasu, Structural characterization of polycrystalline $\mathrm{Ge}$ thin films on insulators formed by diffusion-enhanced Al-induced layer exchange, Japanese Journal of Applied Physics 53 (4S) (2014) 04EH03 (2014). doi:10.7567/jjap.53.04eh03.

32] D. Pelati, O. Mauguin, L. Largeau, F. Brisset, F. Glas, F. Oehler, Kinetics and crystal texture improvements on thin germanium layers obtained by aluminium induced crystallization using oxygen plasma, Surface and Coatings Technology 343 (2018) 121126 (2018). doi:10.1016/j.surfcoat.2017.10.034.

[33] D. V. Gestel, I. Gordon, A. Verbist, L. Carnel, G. Beaucarne, J. Poortmans, A new way to selectively remove Si islands from polycrystalline silicon seed layers made by aluminum-induced crystallization, Thin Solid Films 516 (20) (2008) 6907-6911 (2008). doi:10.1016/j.tsf.2007.12.122.

[34] D. Pelati, G. Patriarche, O. Mauguin, L. Largeau, L. Travers, F. Brisset, F. Glas, F. Oehler, GaAs (111) epilayers grown by $\mathrm{MBE}$ on Ge (111): Twin reduction and polarity, Journal of Crystal Growth 519 (2019) 84-90 (2019). doi:10.1016/j.jcrysgro.2019.05.006.

[35] P. Pukite, P. Cohen, Suppression of antiphase domains in the growth of GaAs on $\mathrm{Ge}(100)$ by molecular beam epitaxy, Journal of Crystal Growth 81 (1-4) (1987) 214-220 (1987). doi:10.1016/0022-0248(87)90393-9.

[36] R. M. Sieg, S. A. Ringel, S. M. Ting, E. A. Fitzgerald, R. N. Sacks, Anti-phase domain-free growth of GaAs on offcut (001) Ge wafers by molecular beam epitaxy with suppressed Ge outdiffusion, Journal of Electronic Materials 27 (7) (1998) 900-907 (1998). doi:10.1007/s11664-998-0116-1.

[37] S. Koh, T. Kondo, Y. Shiraki, R. Ito, GaAs/Ge/GaAs sublattice reversal epitaxy and its application to nonlinear optical devices, Journal of Crystal Growth 227-228 (2001) 183-192 (2001). doi:10.1016/s0022-0248(01)00660-1.

[38] Q. Li, J. L. Krauss, S. Hersee, S. M. Han, Probing interactions of Ge with chemical and thermal $\mathrm{SiO} 2$ to understand selective growth of $\mathrm{Ge}$ on Si during molecular beam epitaxy, The Journal of Physical Chemistry C 111 (2) (2007) 779-786 (2007). doi:10.1021/jp062966o.

[39] S. Plissard, G. Larrieu, X. Wallart, P. Caroff, High yield of selfcatalyzed GaAs nanowire arrays grown on silicon via gallium droplet positioning, Nanotechnology 22 (27) (2011) 275602 (2011). doi:10.1088/0957-4484/22/27/275602.

[40] A. M. Munshi, D. L. Dheeraj, V. T. Fauske, D. C. Kim, J. Huh, J. F. Reinertsen, L. Ahtapodov, K. D. Lee, B. Heidari, A. T. J. van Helvoort, B. O. Fimland, H. Weman, Positioncontrolled uniform GaAs nanowires on silicon using nanoimprint lithography, Nano Letters 14 (2) (2014) 960-966 (2014) doi:10.1021/nl404376m.

[41] Y. Kajikawa, Y. Son, H. Hayase, H. Ichiba, R. Mori, K. Ushi- rogouchi, M. Irie, Suppression of twin generation in the growth of GaAs on Ge (111) substrates, Journal of Crystal Growth 477 (2017) 40-44 (2017). doi:10.1016/j.jcrysgro.2016.12.062.

[42] D. Hull, D. J. Bacon, Introduction to dislocations (Fifth Edition), Butterworth-Heinemann, 2011 (2011). doi:10.1016/B978-0-08096672-4.00019-0.

[43] T. Sadoh, J.-H. Park, R. Aoki, M. Miyao, Low-temperature (300C) formation of orientation-controlled large-grain (10micron) ge-rich $\mathrm{SiGe}$ on insulator by gold-induced crystallization, Thin Solid Films 602 (2016) 3-6 (2016). doi:10.1016/j.tsf.2015.10.057. 